\title{
Predictive diagnostics and personalised medicine in paediatrics
}

\author{
BRAIN, RARE NEUROLOGICAL DISEASES AND \\ EUROPEAN HEALTH CARE SYSTEMS \\ Bowis $J$ \\ OBE, UK
}

"We are here to build a partnership for rare diseases and for the children, in particular, who live with them," and the European Commission should be applauded for its willingness to listen and respond.

A rare disease, by definition, does not affect many people. The paradox is that, as a group, rare diseases are common; there are about 6,000 of them. In the UK, it is estimated that 3.5 million people live with a rare disease. In Europe it is approximately 33 million. Some $75 \%$ of rare diseases affect children, and $30 \%$ of such children die before their fifth birthday (http://www.raredisease.org.uk/index.html). Many of these rare disorders are neurological, it is important for us to recognize that neurological disorders are not exclusively linked to longevity, and that they affect large numbers of children.

For children with neurological disorders we need research into the various causes and the impact of these conditions, as well as potential treatments. This is expensive. It has never been a more difficult time to promote investment in health as a priority for governments, and yet never has there been a more important time to do so. The number of patients affected by rare neurological diseases during childhood makes it clear that these conditions should figure in the mainstream economic argument. If governments and directorates, and international and national agencies, and health services do nothing, the cost of such inaction will be immense. If we invest in research, prevention, treatment and care now, then the future economic benefits can also be immense for individuals, families and economists. We will really personalised treatment and cure patients at the right moment with the adequate amount of drug. For all these reasons, we have to educate the public about these rare disorders and recruit them to the battle to persuade governments and international agencies that child brain research deserves a higher priority and greater investment.

BIRTH ASPHYXIA AS THE MAJOR COMPLICATION IN NEWBORNS: MOVING TOWARDS IMPROVED INDIVIDUAL OUTCOMES BY PREDICTION, TARGETED PREVENTION AND TAILORED MEDICAL CARE

Golubnitschaja $O^{1}$, Yeghiazaryan $K^{1}$, Cebioglu $M^{1}$, Morelli $M^{2}$, Morales $P^{4}$, Herrera-Marschitz $M^{3,4}$

${ }^{1}$ Department of Radiology, Rheinische Friedrich-WilhelmsUniversity of Bonn, Germany

${ }^{2}$ Centre of Excellence for Neurobiology of Dependence, Department of Toxicology, University of Cagliari, Italy ${ }^{3}$ Department of Pharmacology, Karolinska Institute, Sweden

${ }^{4}$ Programme of Molecular \& Clinical Pharmacology, University of Chile, Santiago, Chile

\section{Perinatal asphyxia}

Oxygen deficit at delivery can lead to severe hypoxic ischemic organ damage in newborns followed by a fatal outcome or severe life-long pathologies. The severe insults often cause neurodegenerative diseases, mental retardation and epilepsies. The mild insults lead to so-called "minimal brain-damage disorders" such as attention deficits and hyperactivity, but can also be associated with the development of schizophrenia and life-long functional psychotic syndromes. Asphyxia followed by re-oxygenation can potentially lead to development of several neurodegenerative pathologies, type 2 diabetes mellitus and cancer. 
Birth asphyxia as the major pathology amongst perinatal complications

According to the statistical data collected in years 2000-2002 by the Global Burden of Disease Study, worldwide 56 million deaths occur every year, from that 10.5 million, i.e. $20 \%$ represent children aged below 5 years. In this group, the leading cause of death is perinatal complications. Worldwide epidemiological data demonstrate the perinatal morbidity comprising $60 \%$ of the child death. These facts reflect extensive fieldrelated problems in current healthcare and massive deficits in both targeted prevention of perinatal complications and effective treatments during early postnatal period.

Particularly alarming statistical data are currently delivered by developing countries with large populations (Fig. 1).

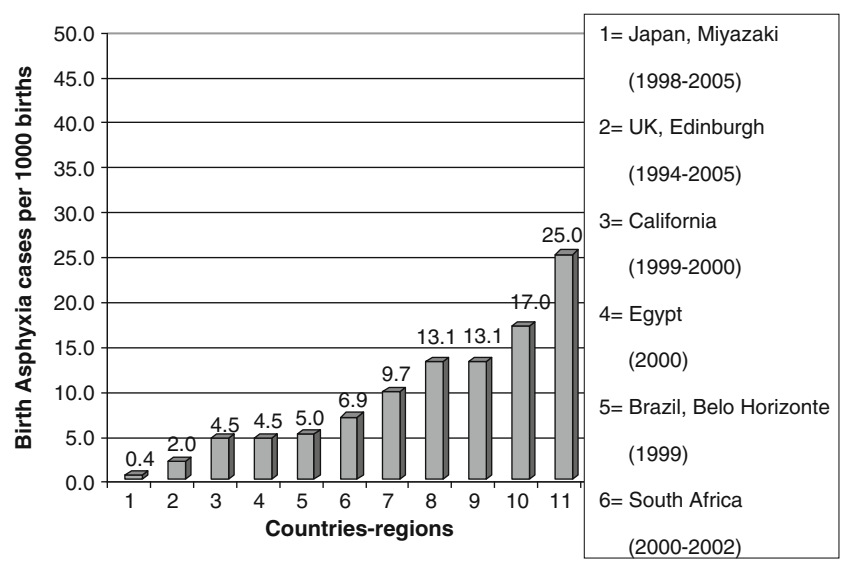

Fig. 1. The prevalence of birth asphyxia as documented by a series of population studies: a corresponding number of cases has been calculated per 1,000 life births. Data taken from [1]

\section{Ethical and socio-economical impacts of educational measures in prevention of birth asphyxia}

Current limited perinatal and postnatal diagnostics cause long-term consequences in a society, which can include a spectrum of negative aspects. From a social and ethical point of view, innovative technologies for an early/ predictive diagnostics and individualised treatment should be applied in all routine medical services. From an economic point of view, emphasis should be put into the costs effectiveness to promote advanced healthcare with appropriate budgets for targeted prevention applied before pathologies manifest.
What are the potential measures that can be applied already now? One example of the high impact of educational measures to improve the healthcare system is given in Fig. 2.

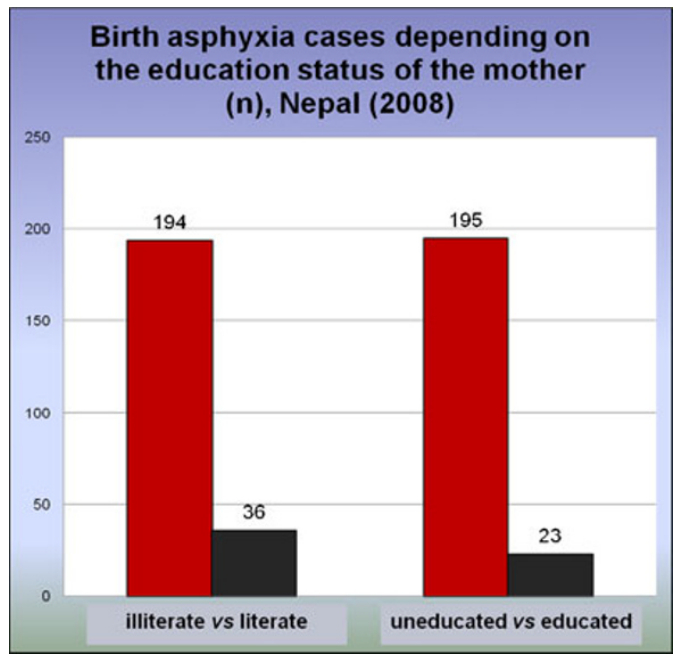

Fig 2. Ratio of newborns with asphyxic deficits born to uneducated/ illiterated versus educated/literated mothers. Data taken from [2]

The prevalence of birth asphyxia registered for children born to educated mothers is several times lower compared with those born to uneducated/illiterate mothers. It is evident that strong restrictions in the amount of education lead to dramatic deficits and costs that are essentially then felt in other branches of the system, including a consequently chaotic healthcare in the society. When the whole spectrum of asphyxia-related pathologies is considered such as type 2 diabetes, neurodegenerative diseases and cancer, the consequent costs increase dramatically for treatment of manifesting pathologies. Therefore, new guidelines are essential to regulate the field in favour of educational measures, personalised treatment approaches before manifesting pathologies, particular emphases on primary prevention, innovative reimbursement programmes introduced by policy-makers.

Expression profiling by "Gene Hunting”- technology discovered functional groups of genes involved in the pathophysiology of perinatal asphyxia

This strategy has been chosen to identify functional groups of genes, differentially regulated in the brain under the severe birth asphyxia versus normoxia. The transcription 
profiling revealed the following functional groups of genes affected by the asphyxia:

- synthesis of signaling molecules (such as nitric-oxide) and heat-shock proteins

- nuclear and DNA-binding factors

- transcription and translation regulation

- energy metabolism

- membrane transport

- ion-handling

- several metabolic pathways (e.g. nucleic acid, lipid metabolism)

- redox-control and free-radical production

- proto-oncogenes.

\section{Blood biomarker-candidates specific for asphyxia related complications}

Blood tests is a highly attractive approach for performing noninvasive examinations to accurately diagnose a severity grade of birth asphyxia and to predict/prognose individual predispositions to or a progression of secondary complications in asphyxia affected newborns. A blood-brain barrier permeability discovered in hypoxic-ischemic excephalopathy well explains potential correlations in pathology specific molecular profiles between brain and blood, justifying the high prognostic value of non-invasive blood examinations.

\section{Outlook}

The task of individual prediction, targeted prevention and personalized treatments before a manifestation of the life-long chronic pathologies usually developed by newborns with asphyxic deficits, should be given the extraordinary priority in neonatology and paediatrics. In the clinical setting, after resuscitation of an infant with birth asphyxia, the emphasis is on supportive therapy. Several interventions have been proposed to attenuate secondary neuronal injuries elicited by asphyxia. Hypothermia has been pointed out to be an effective intervention against the secondary neuronal injury, elicited by the birth asphyxia. Applied immediately after birth asphyxia, hypothermia generally lowers metabolic rates, and diminishes the glutamate levels in brain. Although promising, the clinical efficacy of hypothermia has not been fully demonstrated. It is evident that new approaches are warranted.

In the context of neuro- and anticancer-protection, several sentinel proteins have been described to protect the integrity of the genome (e.g. PARP-1; XRCC1; DNA ligase III $\alpha$; DNA polymerase $\beta$, ERCC2, DNA-dependent protein kinases). They act by eliciting metabolic cascades leading to (i) activation of cell survival and neurotrophic pathways; (ii) early and delayed programmed cell death, and (iii) promotion of cell proliferation, differentiation, neuritogenesis and synaptogenesis. It is proposed that sentinel proteins can be used as markers for characterising longterm effects of perinatal asphyxia, and as targets for novel therapeutic development and innovative strategies for neonatal care [3].

\section{References}

1. Golubnitschaja O, Yeghiazaryan K, Cebioglu M, Morelli M, Herrera-Marschitz M. Birth asphyxia as the major complication in newborns: Moving towards improved individual outcomes by prediction, targeted prevention and tailored medical care. EPMA J. 2011;2: 197-210

2. Golubnitschaja O, Costigliola V. Common origin but individual outcomes: Time for new guidelines in personalised healthcare. Per Med. 2010;7:561-8.

3. Golubnitschaja O. Predictive Diagnostics and Personalized Treatment: Dream or Reality. New York: Nova Science Publishers, 2009.

\section{APPLICATION OF PROTEOMICS FOR THE IDEN- TIFICATION OF PROTEINS WITH DIAGNOSTIC AND THERAPEUTIC IMPORTANCE IN TURNER SYNDROME PATIENTS}

Anagnostopoulos $A K^{1}$, Kolialexi $A^{2}$, Mavrou $A^{2}$, Tsangaris $G T^{l}$

${ }^{1}$ Proteomics Research Unit, Center of Basic Research II, Biomedical Research Foundation of the Academy of Athens, Athens, Greece

${ }^{2}$ Department of Medical Genetics, Athens University School of Medicine, Athens Greece

Turner syndrome (TS) is the most common chromosomal abnormality in females, affecting 1:2500 live female births and is the result of absence or structural abnormality of one $\mathrm{X}$ chromosome. In the present study proteomic techniques were applied in order to identify proteins differentially expressed in the peripheral blood of TS patients as compared to normal females. Plasma samples from 12 girls with TS and a 45, XO karyotype aged from 5 to 7 years and 12 matched for age chromosomally normal controls were analyzed by twodimensional gel electrophoresis (2D-E) followed by matrix assisted laser desorption ionization time-of-flight mass spectrometry (MALDI-TOF-MS). Through densitometric analysis, gel comparison of Turner and normal cases revealed significant differences in protein expression. Eleven proteins; ZA2G, A2GL, OTUB2, FETUA, KNG1, VTDB, APOH, A2MG, FIBA, FIBB and CLUS were up-regulated in Turner syndrome patients whilst four, ACTB, ACTG, HPT and APOE, were found to be down- regulated. Amongst the proteins of interest, a brain specific cell adhesion molecule neurogelin 3 (NL3) was 
noted to adhere differential expression in all samples included in the study. Protein levels of NL3, KNG1, FETUA and APOE were further evaluated by immmunoblot analysis. Detection of differentially expressed molecules could improve our understanding over the unique pathophysiology of the syndrome and allow better management of TS patients.

\section{A NON-INVASIVE FETAL RHD GENOTYPING TEST} TO TARGET ANTENATAL ANTI-D PROPHYLAXIS Tounta $G^{1}$, Kolialexi $A^{1}$, Vrettou $C^{l}$, Papantoniou $N^{2}$, Tsangaris $G^{3}$, Antsaklis $A^{1}$, Kanavakis $E^{1}$, Mavrou $A^{1}$

${ }^{1}$ Department of Medical Genetics, Athens University School of Medicine, Athens, Greece

${ }^{2} 1$ st Department of Obstetrics \& Gynecology, Athens University School of Medicine, Athens, Greece

${ }^{3}$ Proteomics Research Unit, Center of Basic Research II, Biomedical Research Foundation of the Academy of Athens, Athens, Greece

Objective: To design a protocol for non-invasive prenatal diagnosis (NIPD) of foetal Rhesus D (RhD) status.

Methods: 112 single lymphocytes were used to test the efficiency of the assay. The protocol was validated using blood samples from $84 \mathrm{RhD}$-negative pregnant women at 7-24 weeks of gestation.

Isolated cell free DNA (cfDNA) was enzymatically digested using $A c i$ I and analyzed by a multiplex PCR that allowed for the simultaneous amplification of $R H D$ exons 7 and 10, SRY, RASFF1A and ACTB. Amplification of these loci detected fetal $\mathrm{RhD}$ status and gender, confirmed the presence of cfDNA and allowed for assessment of digestion efficiency.

Results: On the one genome equivalent level, the efficiency of the protocol was $\geq 94.6 \%$ for each locus amplified. Conclusive results from the 1st set of PCRs were obtained for 79 cases (94\%) with one false-positive. In 14 samples with negative $R H D$ and $S R Y$ results, the presence of cffDNA was confirmed by amplification of RASSF1A. In 5 cases analysis was repeated and all were accurately diagnosed.

Conclusion: After exclusion of the case with the suspected RHD variant allele, 82/83 samples were accurately diagnosed, with a $3.3 \%$ false positive rate, giving a specificity $96.7 \%$ and $100 \%$ sensitivity.

\section{EVALUATION OF NEURODEGENERATIVE} DISORDERS: THE SEARCH FOR BIOMARKERS

Haseloff $R F^{1}$, Ausseil $J^{2}$, Blasig IE ${ }^{1}$, Heard $J^{3}$

${ }^{1}$ Leibniz-Institut für Molekulare Pharmakologie, BerlinBuch, Germany

${ }^{2}$ Laboratoire de Biochimie, INSERM ERI12, Centre Hospitalier Universitaire d'Amiens, France

${ }^{3}$ Pasteur Institute, Paris, France
To characterize the progress of neurodegenerative diseases, specific biomarkers are needed. The cerebrospinal fluid (CSF) directly contacts the CNS and, hence, represents a stable reporter compartment for the physiological, pathological and pharmacological status of the degenerative brain. The CSF is characterized by a relatively low number of proteins and well suitable for proteomic analyses. The analytics of the potentially more predictive low abundant proteins is complicated by high abundant proteins, such as albumins or globulins, representing more than $75 \%$ of the protein content of the CSF. Therefore, a major problem of CSF proteomics is depletion of the high concentration proteins. We report about our methodical efforts to deplete the major proteins using CSF of different species. Further, we analysed the CSF of a dog model for the neurodegenerative lysosomal storage diseases mucopolysacchrosidosis III B (Sanfilippo syndrome type B) and mucopolysacchrosidosis I (Hurler syndrome). Alterations in the protein pattern, due to the disorders compared to the healthy control, will be discussed to identify candidate biomarkers. Then, clinical studies are required to investigate the transferability of the candidates to the human disease, and to validate them as surrogate markers for MPSI and MPSIIIB.

\section{UNDERSTANDING PATHOGENESIS: FUNDAMENTAL INSIGHTS AND NEW THERAPEU- TIC OPPORTUNITIES}

Platt $F$

University of Oxford, UK

Lysosomal storage disorders occur at a collective frequency of 1:5000 live births and are the commonest cause of paediatric neurodegenerative disease.

Over 50 individual inherited monogenic disorders are known that result from mutations in genes that play a role in multiple aspects of lysosomal function.

The lysosome is the major site of macromolecule degradation/recycling within cells and when lysosomal function is impaired molecules build up over time leading to "storage". The majority of these diseases involve pathology in the brain and are currently without effective therapy.

Despite their simple genetic cause, the gene defect triggers a remarkably complex set of down stream changes within cells/organs/tissues that is termed a "pathogenic cascade". This typically culminates in severe neurological impairment and visceral manifestations. It is interesting to note that aspects of the pathogenic cascade are convergent between lysosomal disorders and more common neurodegenerative diseases.

We are currently in an era of research that is helping tease apart the pathogenic cascade. The aim is to better understand the disease process and identify biomarkers and therapeutic intervention points for clinical translation. 
A particularly exciting aspect of this research is it may identify clinical intervention points that could be treated with currently available/approved drugs speeding up the translational process. Due to the intrinsic heterogeneity of these diseases personalised medicine will be required to maximise clinical benefit.

\section{TREASURING EXCEPTIONS: GOING FROM THE PERSONAL TO THE FUTURE FOR ALL}

Cox TM

Department of Medicine, University of Cambridge, UK

Fully completed in 2003, the lexicon of the human genome stands at an epoch in medical research. This unprecedented collaboration has brought innovative sequencing technologies and bioinformatic analysis to bear on intractable problems in human genetics - not least of which has been the discovery and characterization of hitherto unknown disease-related genes.

Today, the structure of about 6,000 genes implicated in human diseases has been elucidated; this includes many that cause rare disorders. Such rare ('orphan' or even 'ultra-orphan') conditions have long been known, but it is only now that they can be annotated and characterized - and understood at the molecular level. Moreover, in these times of relative affluence, even striking scientific discoveries have been accompanied by a societal revolution in which there is a collective desire to understand the burden that rare disabilities represent to those affected, as well as their immediate families.

With a rational perception of hereditary illness through 'personalised genetic health' has come the realization that disease not only places a physical burden on society but an imperative to assume the moral burden of responsibility for its relief. The view of the diseased person as a broken machine, with damaged parts, or even the whole, to be replaced (or, in the case of some hereditary diseases, to be disposed of), is outmoded. In this respect, the Darwinian view is both rational and philanthropic: as Archibald Garrod showed, much illness is the product of interactions between the individual and an environment to which they are uniquely maladapted at a particular time. The constitutional factors that come together in the individual patient are the result of selective or neutral factors introduced over millennia persist as complex determinants of the existence of our species.

We are at the zenith of a golden age in which discoveries in biology are being rapidly translated into improved medicines and, even with the most intractable diseases such as those that injure the brains of infants and children, therapeutic avenues are opening up. Advances in cellular and molecular biology allow us to have a comprehensive understanding of causation - but they also provide the basis for our search for cures. Challenges remain however - and ensuring that therapeutic quantities of corrective medicines reach the injured brain is a hurdle that merits intensive effort.

Here I discuss exceptional diseases as a paradigm for discovery and as an unusual pathway in basic and applied research. The conflation of orphan drug legislation and the emerging power and commitment of patient organisations can synergise to advance basic research and therapeutic development in ultra-orphan diseases. In many instances gene transfer and the emerging stem-cell techniques offer more immediate promise of definitive correction of otherwise devastating disorders, such as those which affect the brain; but their therapeutic development and clinical application is beset with regulatory difficulties and lack of political commitment.

Overcoming these impediments is mandatory for realizing the value of our scientific investment- already it offers potential for the relief of rare diseases that affect the brain of the young and vulnerable. But this call has another critical dimension, and one founded on a longstanding and unassailable principle of medical science - that understanding rare inherited diseases provides unique insights into the therapy of more common ones. There are many rare and disabling diseases: as a group not only do they constitute an unquantifiable individual personal burden to patients and their families, but they are prevalent. Moreover, as a consequence of the natural genetic variation that maintains our species, rare inherited diseases are a treasury for discovery and biological progress. If we are to reduce their personal burden, we must invest in research to understand their secrets and at the same time invest directly to improve our future.

\section{PATIENT-CENTRIC THERAPY AT THE RIGHT MOMENT: CHALLENGES AND OPPORTUNITIES IN THE DEVELOPMENT OF TREATMENTS FOR CHILDREN WITH RARE NEUROLOGICAL DISORDERS}

Barton NW

Shire Human Genetic Therapies, Cambridge, MA, USA

The development of effective therapies for children with inherited neurological diseases is a well recognized need given the cumulative public health impact of these disorders. Collaboration of multiple stakeholders including academic scientists and physician investigators, patient advocacy organizations, funding authorities and regulatory bodies is required in order to make substantive progress toward this goal. Selection of a disease target with a clear therapeutic approach and committed investment by concerned parties are key steps early in the process. Demonstration of the potential benefits of a proposed therapy in a relevant animal model is typically required to attract a suitable biopharmaceutical partner who will have the resources to advance the project further in development. From a biopharmaceutical perspective, there are several 
unique challenges inherent in the development of personalized medicines particularly for children with rare brain diseases. Frequently the natural history of these diseases is poorly understood and few patients are available for clinical study. To further compound matters, correlations between the genetic defect and the clinical features of the disease are often not clear. An additional, frequently encountered limitation is the small number of experts with deep clinical knowledge of the illness of interest. Once the decision has been taken to advance a compound with a supporting toxicology package into clinical development, one must interact with regulatory bodies that may be unfamiliar with the illness and what body of evidence constitutes proof of benefit. These challenges are often amplified by the lack of accepted clinical endpoints and known biomarkers that inform dose selection. Given the limited number of affected children who can participate in clinical studies of rare neurodegenerative disorders, late stage clinical trials must be executed on a global scale in order to complete trial enrollment in a timely manner. Clearly to succeed in the face of these risks and challenges, substantial investment is required by all parties: governmental authorities, advocacy groups and industry. Given all of this complexity, what is the opportunity? Success responds to huge unmet human need and provides benefits to all.

ANTI-STREPTOLYSIN O (ASO) TITER AND ANTI-BASAL GANGLIA ANTIBODIES (ABGA) IN A LARGE COHORT OF TOURETTE PATIENTS: POSSIBLE PERSPECTIVE OF MORE TARGETED PREVENTIVE AND THERAPEUTIC APPROACHES Rizzo $R^{1}$, Chiarotti $F^{2}$, Buttiglione $M^{3}$, Cardona $F^{4}$, Gulisano $M^{1}$, Nardocci $N^{5}$, Orefici $G^{2}$, Veneselli $E^{6}$, Vitiello $F^{3}$, Martino $D^{8}$

${ }^{1}$ Child and Adolescent Neuropsychiatry, Materno-Infantile and Radiological Sciences Department, University of Catania, Italy

${ }^{2}$ Istituto Superiore di Sanità, Rome, Italy

${ }^{3}$ Dept. of Human Pharmacology and Physiology, Univ. of Bari, Italy

${ }^{4}$ Department of Child and Adolescent Neuropsychiatry, University of Rome "La Sapienza", Rome, Italy

${ }^{6}$ Besta Neurological Institute, Milan, Italy

${ }^{7}$ Istituto G. Gaslini, Genoa, Italy

${ }^{8}$ Dep. of Neurol. and Psych. Sciences, Univ. of Bari, Italy

Introduction: Tourette syndrome (TS) is a childhood-onset neuropsychiatric disorder characterized by multiple motor and vocal tics and high co-morbidity rates with obsessive compulsive disorder (OCD), attention deficit and hyperactivity disorder (ADHD) (Swain et al., 2007). Detracting further from the quality of life of affected individuals, TS patients show deficits in procedural learning and have an increased risk for depression and anxiety (Marsh, 2004). The etiology and the pathophysiology of TS remain poorly understood. It is currently thought that environmental and genetic factors interact in order to lead to the onset of symptoms. However, the exact role and the level of contribution of each of these factors have not been yet elucidated (State, 2011).

Among environmental factors, the possible involvement of Group A Streptococcus (GAS) infection has been proposed, mainly following the description of a rare group of TS patients in whom tics and behavioural features fluctuate in association with variations in the immunological response to GAS [the so-called Paediatric Autoimmune Neuropsychiatric Disorders Associated with Streptococcal infection (PANDAS)] (Sweedo et al. 1998). Nevertheless, widely accepted consensus-based criteria for the definition of PANDAS are currently lacking.

Irrespective of the controversy concerning the concept of PANDAS, preliminary but consistent data support the presence of abnormalities of immune regulation in children with TS (Kawikova et al. 2007). These alterations suggest that environmental factors, among which GAS infections, active on the immune system could modify the course of severity of tics and associated symptoms in TS.

Aims: To evaluate the relationship between diagnosis and clinical course of Tourette syndrome (TS) and exposure to Group A Streptococcus (GAS).

Methods: GAS pharyngeal infections, anti-streptococcal antibody response and expression of anti-basal ganglia antibodies (ABGA) were compared between 168 TS patients (mean $\pm \mathrm{SD}$ age, 10 years 2 months \pm 31 months) and 177 control patients with epileptic or sleep disorders (mean \pm SD age, 9 years, 8 months \pm 34 months), recruited in four Italian tertiary referral outpatient services. Onehundred-and-forty-four TS patients were followed up at 3monthly intervals for a mean of 3.3 visits/patient; exacerbations of tics, obsessive-compulsive symptoms and symptoms related to attention deficit/hyperactivity disorder, depression and anxiety were defined by a bootstrap procedure using validated rating scales. We assessed the effect of new GAS infections and new ABGA identification upon the risk of exacerbations using logistic regression analysis. Odds ratio (OR) were computed on each type of exacerbation adjusted for age, inter-visit interval, and medication changes. All statistical analyses were performed using statistical software package (Stata 11).

Results: When analyzed cross-sectionally, patients with TS exhibited, compared to controls, a higher frequency of GAS throat infection $(7.7 \%$ vs. $1.7 \% ; p=0.009)$, pathological anti-streptolysin O (ASO) titers $(57.7 \%$ vs. $33.9 \% ; p<$ $0.0001)$, and ABGA $(25.3 \%$ vs. $8.2 \% ; p<0.0001)$. The prospective study showed that ASO titers were persistently 
elevated in $57 \%$ of TS patients; however, new GAS infections or newly identified ABGA did not significantly predict the occurrence of either type of exacerbations of obsessive-compulsive, ADHD, depressive or anxiety symptoms (table 1).

Interpretation: TS patients might be more prone to GAS infections and develop stronger antibody responses to GAS, probably as a result of underlying immune dysregulation. At the same time, new GAS infections are unlikely to exert, years after the onset, a relevant effect upon the severity of neuropsychiatric symptoms.

COMPARATIVE STUDY OF ALLELE FREQUENCIES OF PAI-1, MTHFR, FV, FI AND GPIIIA GENE POLYMORPHISMS IN CHILDREN WITH HENOCH-SCHÖNLEIN PURPURA, SCHAMBERG DISEASE AND HEALTHY CHILDREN

Shirinbekova NV, Voronina EV, Vitovich AN, Larionova VI

St. Petersburg State Pediatric Medical Academy, St. Petersburg, Russia

Objectives: Comparative analysis of alleles and genotypes distribution of plasminogen activator inhibitor 1 (PAI-1) $6754 G / 5 G$ insertion-deletion polymorphism, methylenetetrahydrofolate reductase (MTHFR) $-677 C / T$ polymorphism, blood coagulation factor $V$ (FV) $1691 \mathrm{G} / \mathrm{A}$ polymorphism, blood coagulation factor $I(F I)-455 G / A$ polymorphism and platelet GpIIb/IIIa Pl A1A2 polymorphism in (1) children with Henoch-Schönlein purpura (HSP), (2) in children with Schamberg Disease (SD) and (3) in healthy children.

Patients and methods: HSP group consisted of 40 patients (20 boys and 20 girls), SD-group consisted of 30 patients (18 boys and 12 girls). Control group was composed of 46 apparently healthy children (22 boys and 24 girls). PAI-1$6754 G / 5 G$ insertion-deletion polymorphism was analysed by PCR method, MTHFR 677C/T, FV 1691G/A, FI -455G/ $A$ and GpIIIa $\mathrm{Pl} \mathrm{AlA2}$ polymorphisms were analysed by PCR-RFLP method. Statistical significance of differences between groups was assessed using $\chi^{2}$ tests.

Results: There was no statistically significant $(p>0.05)$ difference in allele and genotype distribution of analysed polymorphisms between HSP group, SD group and control group. The genotype distribution was in accordance with Hardy-Weinberg equilibrium for all variants. Genotype $5 G /$ 5G PAI-1-675 wasn't found in SD group. Genotype PlA2A2 GpIIIa wasn't found in HSP and control groups. 1691A allele of the $F V$ gene wasn't found in any group (all children has "normal" $G G$ genotype $F V 1691$ ).

Conclusion: In this study we did not find PAI-1-675 5G/5G genotype in children with $\mathrm{SD}$ and there was no $A$-allele $F V$ $1691 G / A$ in any of studied groups. Genotype PlA2A2 GpIIIa was found neither in HSP, nor in control groups.
THE PERSONALIZED APPROACH TO DRUG DOSING OF VALPROIC ACID AT WOMEN WITH EPILEPSY, WHO ARE CARRIERS OF MUTATIONS OF FOLATE CYCLE GENES

Dmitrenko D, Pilugina M, Shnayder N

Krasnoyarsk State Medical University named after Prof. V.F. Vojno-Jasenetsky, Krasnoyarsk, Russia

The importance of the personalized approach to valproic acid dosing at childbearing age women with epilepsy is determined by increased risk of developing secondary disorders of folate cycle and consequence of impaired fertility function. This is particularly important for patients who are carriers of certain gene polymorphisms of folate cycle: for MTHFR gene- polymorphic variants c.665 C $>\mathrm{T}$ (Ala222Val) and c.1286 A $>$ C (Glu429Ala), for MTR genepolymorphism c.2756 $\mathrm{A}>\mathrm{G}$ (Asp919Gly), and for MTRR gene - c.66 polymorphism $A>G$ (Ile22Met). The combination of homozygous variant carriers of c. 665 polymorphism $\mathrm{C}>\mathrm{T}$ (Ala222Val) (T/T) and homozygous variant polymorphism c.1286 A $>$ C (Glu429Ala) (A/A) of MTHFR gene leads to significant reduction of folic acid in blood serum. In addition the administration of anti-epileptic drugs (VPA aggravates epilepsy) may lead to adverse drug events (ADE) in form of impaired fertility (menstrual disorders, miscarriage, increased risk of congenital malformation of neural tube and chromosomal abnormalities in a child despite normal karyotype of parents). These ADE may have long-term consequences, even in the case of administration of sub-therapeutic doses of VPA.

The risk of ADE increases by homozygous variant polymorphism of MTR $(\mathrm{G} / \mathrm{G})$ and/or heterozygous variant $(\mathrm{A} / \mathrm{G})$. The influence of this polymorphism on potentially impaired fertility can be compounded by increased homocysteine levels in carriers of MTHFR-related polymorphisms, even without additional supplementation of VPA. There are also high impacts of the prevalent heterozygous variant gene polymorphism in MTR $(\mathrm{A} / \mathrm{G})$ in the population. At least 3 months before a planned pregnancy and during the 1st trimester of it, an individualised diet by enrichment of folic acid and vitamin B12 is proposed for women, who are carriers of homozygous and heterozygous MTR polymorphisms - c.2756 A $>\mathrm{G}$ (Asp919Gly), especially when combined with MTHFR polymorphisms - c.665 C $>\mathrm{T}$ (Ala222Val) and/or c.1286 A $>$ C (Glu429Ala).

An impaired fertility is ten-time more frequent in women with epilepsy under long-term VPA administration, who are carriers of MTHFR or/and MTR and CYP2C9*2 and CYP2C $9 * 3$ polymorphisms. Detection of these polymorphisms among women with epilepsy is clinically relevant, since they require initial selection of individual low-dose VPA medicamentation. In such cases the standard drug- 
dosages can lead to excessive accumulation of VPA in serum and to extension of its metabolism in the liver resulting in lower quality of life of young women by fertile dysfunction. "Down-stream" complications can be further expected such as a social and family maladjustment, development of anxiety-depressive syndrome, disintegration of family.

\section{DRUGS MAY CONTRIBUTE TO THE APPEAR-} ANCE OF ORAL-FACIAL CLEFTS IN NEWBORNS Velickova N, Gacova M, Kamcev N, Angelovska B, Dimova $C$

Faculty of medical science, University "Goce Delcev"Stip, Macedonia

Introduction: A cleft is a gap in a body structure that results from incomplete closing of a specific structure during development. Clefts that occur in the lip and palate (roof of the mouth) are called oral-facial clefts. These defects occur very early during fetal development. Cleft lips and palates may be caused by a combination of genetic and environmental factors.

The aim of this study was to evaluate potential risks factors in children hospitalized in the faculty hospital of Stip for effective preventive measures reducing an appearance of oral-facial clefts in newborns.

Material and methods: Oral-facial clefts were diagnosed by physical exam after delivery. Potential risks factors and drug exposure of mothers during pregnancy were analyzed.

Results: The results indicated that during past 10 year there was an increasing tendency in the incidence rate of oral-facial clefts in newborns. Further, the oral-facial defects were accompanied by other complications such as feeding difficulties, frequent ear infections and hearing loss.

Conclusion: Maternal drug abuse (amoxicillin, phenytoin, oxprenolol, and thiethylperazine taken during the second and third month of pregnancy), smoking, and pesticide exposure were registered as the statistically significant risk factors for oral-facial defects in newborns. 